\title{
Representation of the Matrix for Conversion between Triangular Bezier Patches and Rectangular Bezier Patches
}

\author{
Pembe Sabanctgil ${ }^{1, *}$ and Mustafa Kara ${ }^{1}$ \\ ${ }^{1}$ Department of Mathematics, Eastern Mediterranean University, Gazimağusa, TRNC, Mersin 10, \\ Turkey \\ ${ }^{1}$ Department of Mathematics Eastern Mediterranean University, Gazimağusa, TRNC, Mersin 10, \\ Turkey
}

\begin{abstract}
In this paper we studied Bezier surfaces that are very famous techniques and they are widely used in the area of Computer Aided Geometric Design. Mainly there are two kinds of Bezier surfaces which are classified as rectangular and triangular Bezier patches. In this paper we will give a simple representation for the conversion matrix which converts one type to another type in one step.
\end{abstract}

\section{Introduction}

The theory of Bezier curves has an important role and they are numerically the most stable among all polynomial bases currently used in CAD systems. On the other hand in these days Bezier surfaces are very famous techniques and widely used in Computer Aided Geometric Design (see [1] and [2]). Mainly there are two kinds of Bezier surfaces which are triangular and rectangular Bezier patches and their definition is given by the uni-variate Bernstein polynomials $B_{i}^{n}(s)=\left(\begin{array}{l}n \\ i\end{array}\right) s^{i}(1-s)^{n-i}$ and the bi-variate Bernstein polynomials $B_{i, j, k}^{n}(\alpha, \beta, \eta)=\left(\begin{array}{c}n \\ i, j, k\end{array}\right) \alpha^{i} \beta^{j} \eta^{k}$ where the sum $\alpha+\beta+\eta=1$. Definition of an n-th degree triangular Bezier patch with control points $D_{i, j, k}$ is given by

$$
D(\alpha, \beta, \eta)=\sum_{i+j+k=n} D_{i, j, k} B_{i, j, k}^{n}(\alpha, \beta, \eta), \quad \alpha, \beta, \eta \geq 0, \quad \alpha+\beta+\eta=1 .
$$

On the other hand $n \times m$ degree rectangular Bezier patch with the control points $P_{i, j}$ is defined by

$$
P(\alpha, \beta)=\sum_{i=0}^{n} \sum_{j=0}^{m} P_{i, j} B_{i}^{n}(\alpha) B_{j}^{m}(\beta), 0 \leq \alpha, \beta \leq 1, \quad(\text { see }[3])
$$

\footnotetext{
* Corresponding author: pembe.sabancigil@emu.edu.tr
} 
Since these two patches have different geometric properties, it is not easy to deal with both of them in one Computer Aided Design system (see [4] and [5]). Thus from this reason we have to convert one type to another.

\section{Construction of the Conversion Matrices}

In the following theorem (see [3]), it is given that a triangular Bezier patch can be converted to an equivalent degenerate rectangular Bezier patch, whose control points are computed by degree elevating some Bezier curves. Here a degenerate rectangular patch means a rectangular patch in which one of its edges collapsed into a point.

Theorem 1. (see [3]) A degree $n$ triangular Bézier patch $D(\alpha, \beta, \eta)$ can be represented as a degenerate rectangular Bézier patch of degree $n \times n$ :

$$
P(\alpha, \beta)=\sum_{i=0}^{n} \sum_{j=0}^{n} P_{i, j} B_{i}^{n}(\alpha) B_{j}^{n}(\beta), 0 \leq \alpha, \beta \leq 1
$$

where $P_{i, j}$ are the control points and they are determined as

$$
\left(\begin{array}{c}
P_{i, 0} \\
P_{i, 1} \\
\vdots \\
P_{i, n}
\end{array}\right)=A_{1} A_{2} \ldots A_{i}\left(\begin{array}{c}
D_{i, 0} \\
D_{i, 1} \\
\vdots \\
D_{i, n-i}
\end{array}\right), \quad i=0,1, \ldots, n
$$

and $A_{i}$ where $i=0,1, \ldots n$, are matrices (or operators) of degree elevation which are represented in the following form:

$$
A_{i}=\left[\begin{array}{cccccc}
1 & 0 & 0 & \cdots & 0 & 0 \\
\frac{1}{n+1-i} & \frac{n-i}{n+1-i} & 0 & \cdots & 0 & 0 \\
0 & \frac{2}{n+1-i} & \frac{n-i-1}{n+1-i} & \cdots & 0 & 0 \\
\vdots & \vdots & \vdots & \ddots & \vdots & \vdots \\
0 & 0 & 0 & 0 & \frac{n-i}{n+1-i} & \frac{1}{n+1-i} \\
0 & 0 & 0 & 0 & 0 & 1
\end{array}\right]_{(n-i+2) \times(n-i+1)}
$$


Here in Theorem 2 we give a simple representation for the matrix product $A_{1} A_{2} \ldots A_{i}$.

Theorem 2. The product of the degree elevation matrices that converts a degree $n$ triangular Bezier patch to a degenerate rectangular Bezier patch of degree $n \times n$ is given as follows :

where

$$
A_{1} A_{2} \ldots A_{k}=\bar{A}_{k}=\left[\bar{a}_{i, j}{ }^{(k)}\right]_{(n+1) \times(n-k+1)}
$$

$$
\bar{a}_{i, j}{ }^{(k)}=\frac{\left(\begin{array}{c}
i-1 \\
j-1
\end{array}\right)(k)_{i-j}(n-k)_{j-1}}{(n)_{i-1}}
$$

$$
\begin{gathered}
(k)_{n}=k(k-1) \ldots(k-n+1)=\prod_{j=1}^{n}(k-(j-1)) \quad \text { and } \\
A_{k}=\left[\begin{array}{cccccc}
1 & 0 & 0 & \ldots & 0 & 0 \\
\frac{1}{n+1-k} & \frac{n-k}{n+1-k} & 0 & \ldots & 0 & 0 \\
0 & \frac{2}{n+1-k} & \frac{n-k-1}{n+1-k} & \ldots & 0 & 0 \\
\vdots & \vdots & \vdots & \ddots & \vdots & \vdots \\
0 & 0 & 0 & 0 & \frac{n-k}{n+1-k} & \frac{1}{n+1-k} \\
0 & 0 & 0 & 0 & 0 & 1
\end{array}\right]_{(n-k+2) \times(n-k+1)}
\end{gathered}
$$

Now in the following theorem we will consider the inverse process which will convert a rectangular Bezier patch of degree $n \times n$ to a triangular Bezier patch of degree $n$ :

Theorem 3 A rectangular Bezier patch $P(\alpha, \beta)$ of degree $n \times n$ can be represented as a triangular Bezier patch $D(\alpha, \beta, \eta)$ of degree $n$ :

$$
D(\alpha, \beta, \eta)=\sum_{i+j+k=n} D_{i, j, k} B_{i, j, k}^{n}(\alpha, \beta, \eta), \quad \alpha, \beta, \eta \geq 0, \quad \alpha+\beta+\eta=1
$$


where $D_{i, j, k}$ are the control points and they are defined as

$$
\left(\begin{array}{l}
D_{i, 0} \\
D_{i, 1} \\
\vdots \\
D_{i, n-i}
\end{array}\right)=B_{i} B_{i-1} \ldots B_{1}\left(\begin{array}{c}
P_{i, 0} \\
P_{i, 1} \\
\vdots \\
P_{i, n}
\end{array}\right) i=0,1, \ldots, n
$$

and $B_{i}(i=0,1, \ldots ., n)$ are matrices (or operators) of degree reduction which are given in the following form

$$
B_{k}=\left[\begin{array}{ccccccc}
1-t & t & 0 & 0 & \cdots & 0 & 0 \\
0 & 1-t & t & 0 & \cdots & 0 & 0 \\
0 & 0 & 1-t & t & \cdots & 0 & 0 \\
\vdots & \vdots & \vdots & \ddots & \ddots & \vdots & \vdots \\
0 & 0 & 0 & 0 & 1-t & t & 0 \\
0 & 0 & 0 & 0 & & 1-t & t
\end{array}\right]_{(n-k+1) \times(n-k+2)}
$$

Theorem 4 The product of the matrices in the above theorem $B_{k} B_{k-1} \ldots B_{1}$ that converts a rectangular Bezier patch of degree $n \times n$ to a triangular Bezier patch of degree $n$ can be generalized as follows:

$$
Z^{k}=B_{k} B_{k-1} \cdots B_{1}=\left[\begin{array}{ccccccccccc}
b_{k, 0} & b_{k, 1} & b_{k, 2} & \cdots & b_{k, \mathrm{k}-1} & b_{k, \mathrm{k}} & 0 & 0 & 0 & \cdots & 0 \\
0 & b_{k, 0} & b_{k, 1} & b_{k, 2} & \cdots & b_{k, \mathrm{k}-1} & b_{k, \mathrm{k}} & 0 & 0 & \cdots & 0 \\
0 & 0 & b_{k, 0} & b_{k, 1} & b_{k, 2} & \cdots & b_{k, \mathrm{k}-1} & b_{k, \mathrm{k}} & 0 & \cdots & 0 \\
0 & 0 & 0 & b_{k, 0} & b_{k, 1} & b_{k, 2} & \cdots & b_{k, \mathrm{k}-1} & b_{k, \mathrm{k}} & \cdots & 0 \\
\vdots & \vdots & \vdots & \ddots & \ddots & \ddots & \ddots & \ddots & \ddots & \ddots & 0 \\
0 & 0 & 0 & 0 & 0 & b_{k, 0} & b_{k, 1} & b_{k, 2} & \cdots & b_{k, \mathrm{k}-1} & b_{k, \mathrm{k}}
\end{array}\right]_{(n-k+1) \times(\mathrm{n}+1)}
$$

where $b_{k, j}=\left(\begin{array}{c}k \\ j\end{array}\right) t^{j}(1-t)^{k-j}$

Remark The proofs of Theorems 2, 3 and 4 will be presented later in the complete paper.

\section{Conclusion}

In this paper, we studied on the conversion matrix to convert triangular Bezier patch to a rectangular Bezier patch and a rectangular Bezier patch to a triangular Bezier patch. We found simple representations for these two matrices which will allow the conversion in one step. 


\section{References}

1. Farin, G., Curve and Surface for CAGD : A Practical Guide. Academic Press, New York (1990)

2. Farin, G., Computer Aided Design, 21, 293-296 (1989)

3. Shi-Min Hu, Computer Aided Geometric Design, 18, 667-671 (2001)

4. Goldman, R., Filip, D., Computer Aided Design, 19, 25-27 (1987)

5. Shi-Min Hu, Computer Aided Geometric Design, 13, 219-226 (1996) 